\title{
Recombinant Human Granulocyte Colony Stimulating Factor (Rh G-CSF) Administered Before, During and After High Dose Chemotherapy to Patients with Acute Myeloid Leukemia: Effect on Hematopoiesis and Bone Marrow Microenvironment
}

\author{
Anwarul Islam*
}

Clinical Associate Professor of Medicine, State University of New York at Buffalo. Division of Hematology/Oncology, Department of Medicine, Buffalo General Hospital, Room E 318 Buffalo, New York 14203, USA

*Corresponding author: Anwarul Islam, M.D., Ph.D., FRCPath, FACP, Clinical Associate Professor of Medicine, State University of New York at Buffalo. Division of Hematology/Oncology, Department of Medicine, Buffalo General Hospital, Room E 318 Buffalo, New York 14203, USA

\section{ARTICLE INFO}

Received: 蔧 July 28, 2019

Published: 蔧 August 05, 2019

Citation: Anwarul Islam. Recombinant Human Granulocyte Colony Stimulating Factor (Rh G-CSF) Administered Before, During and After High Dose Chemotherapy to Patients with Acute Myeloid Leukemia: Effect on Hematopoiesis and Bone Marrow Microenvironment. Biomed J Sci \& Tech Res 20(2)-2019. BJSTR. MS.ID.003434.

Keywords: Bone marrow biopsy; Plastic embedding; Acute myeloid leukemia; Hematopoiesis; Hematopoietic Regeneration; Eosinophils; Plasma cells; Fat cells

Abbreviations: BM: Bone Marrow; AML: Acute Myeloid Leukemia; BT: Bone Trabeculae; MPO: Myelo-Peroxidase; HGFs: Hematopoietic Growth Factors; CSFs: Colony Stimulating Factors; ILs: Interleukins; G-CSF: Granulocyte Colony-Stimulating Factor; GM-CSF: Granulocyte-Macrophage Colony Stimulating Factor; GMA: Glycol-Methacrylate; MGG: May-Grunwald-Giemsa; TBS: Tris Buffered Saline; AEC: Amino-Ethylcarbazole

\section{ABSTRACT}

The effects on bone marrow (BM) cell proliferation and differentiation of recombinant human granulocyte colony stimulating factor (Rh G-CSF) administered before, during and after high-dose chemotherapy were studied in six patients with acute myeloid leukemia (AML) who went into complete remission. The changes induced by chemotherapy and cytokine treatment were assessed by morphologic and immunohistochemical analysis of sequential plastic embedded BM biopsy sections. The pre-therapy marrows were very cellular (cellularity range 60-80\%, except for one case where the cellularity was less than $50 \%$ ). Immediately following chemotherapy, the marrows became extremely hypocellular (cellularity ranged from 5-15\%), edematous and contained widely dilated sinuses. The cells now present were diffusely scattered and consisted mostly of lymphocytes, plasma cells, tissue mast cells and macrophages. Plasma cells were mostly seen clustered around the small blood vessels as perivascular cuffs. They were polyclonal as demonstrated by the staining pattern with anti-kappa and anti-lambda light chain antibodies. BM stromal changes included migration of endosteal cells into the nearby vacuous and edematous marrow space as fibroblast-like cells.

Within two to four weeks following the cessation of chemotherapy clusters of regenerating hematopoietic cells were seen adjacent to bone trabeculae (BT), near marrow sinusoids, in close proximity to fat cells, and also surrounding the smaller blood vessels. Later, a proportion of these cells were observed to migrate into the central intertrabecular marrow space where they formed colonies of more mature and differentiated hematopoietic cells. By 5-8 weeks after the completion of the chemotherapeutic protocol, normal (trilineage) hematopoiesis was restored in all patients. Hematopoiesis during the early (2-4 weeks) as well as the late (5-8 weeks) phases of the medullary regeneration was primarily granulocytic. This was confirmed by the presence of increased number of myelo-peroxidase (MPO) positive cells in these areas. Interestingly, BM biopsies obtained 5-8 weeks following the end of chemotherapy and following the cessation of cytokine treatment showed considerable increase in eosinophilic granulocytes even though the number of circulating eosinophils in the peripheral blood remained within normal limits.

The distribution of eosinophils was predominantly perivascular, although a large number of these cells were seen scattered and diffusely spread throughout the sections. G-CSF is not known to cause eosinophilia and we believe this is the first report which shows that the administration of G-CSF may also cause medullary eosinophilia without affecting the eosinophil count in the peripheral blood, at least within the first 8 weeks of observation. It is suggested that the hematopoietic regeneration demonstrated by the granulocytic proliferation was most likely the result of the association of the treatment with Rh G-CSF. Furthermore, the proximity of the regenerating hematopoietic cells to the endosteum, vascular endothelium and fat cells may be indicative of some functional relationships among these cells. 


\section{Introduction}

Hematopoietic growth factors (HGFs) are a group of glycoproteins, termed colony stimulating factors (CSFs) or interleukins (ILs) that are believed to control the proliferation, differentiation and survival of hematopoietic stem and/ or progenitor cells. In addition, they also affect a variety of functional activities of mature and terminal hematopoietic cells [1-4]. A number of recombinant human hematopoietic growth factors, such as granulocyte colony-stimulating factor (G-CSF), granulocyte-macrophage colony stimulating factor (GM-CSF), and interleukin-3 (IL-3) are already proving their usefulness in the treatment of bone marrow (BM) failure caused by chemotherapy, radiotherapy, and various hematopoietic disorders [5-8]. IL-3 acts on the early progenitor pool, i.e, multipotent stem cells such as CFU-GEMM, early erythroid (BFU-E), granulo-monocytic (CFUGM) and megakaryocytic progenitors. GM-CSF exerts similar effects, but possibly stimulates progenitors at more distal stages of differentiation [5-8]. Although the in vivo hematopoietic effects of rhIL-3, Rh GM-CSF have already been documented [9], little has been reported regarding the in vivo activity of Rh G-CSF. In this report we document the effects of Rh G-CSF administered during chemotherapy to six patients with acute myeloid leukemia who responded to treatment and displayed apparent complete morphologic remission within the initial eight-week period of treatment.

\section{Materials and Methods}

Bone-marrow biopsy specimens were obtained from 6 AML patients at different time intervals during the course of intensive remission induction chemotherapy and after its completion. One to six specimens were obtained from each patient during the first 8 weeks of observation. The patients were treated with one course of cytosine arabinoside [(Ara-C) $3 \mathrm{gm} / \mathrm{m}^{2}$ as a 1 hour intravenous infusion q12 hours for 12 doses (days 1-6)] and idarubicin [(Idamycin) $12 \mathrm{mg} / \mathrm{m}^{2}$ IV over a 30 minute period, daily for three days immediately following doses \#3,5 and 7 of Ara-C $(24,48$ and 72 hours)] In addition, each patient also received G-CSF (10 mcg/kg/ day, as a continuous intravenous infusion) before and during chemotherapy, and (10 mcg/kg/day, subcutaneously) after completion of induction chemotherapy for an average of 24 days [10].

BM biopsies were obtained from the posterior iliac crest using a manual trephine $[11,12]$ and were processed into plastic glycol-methacrylate (GMA), without decalcification, according to previously described methods $[13,14]$. Briefly, the biopsies were initially immersed in Bouin's fixative for three hours, dehydrated in graded methanol solutions, and then processed into GMA. Sections were cut at a thickness of $2 \mu \mathrm{m}$, attached to Histostik-coated glass slides (Accurate Chemicals, Westbury, New York) and stained with May-Grunwald-Giemsa (MGG) stain. Antisera to human kappa and lambda light chains and polyclonal rabbit antisera to human myeloperoxidase were obtained from Dako Corporation, Santa Barbara, California. Monoclonal antibody to eosinophil peroxidase was a generous gift from Oncogene Science. The sections were first dipped in a $0.3 \%$ hydrogen peroxide solution in absolute methanol for 20 minutes to inhibit endogenous peroxidase activity.

The sections were then washed three times for 5 minutes each in Tris buffered saline (TBS). The sections were digested with pronase $(0.2 \mathrm{mg} / \mathrm{ml}$ in TBS $)$ for 10 minutes at $37^{\circ} \mathrm{C}$ in a moist chamber and washed in TBS as described above. The non-specific reactivity to the secondary antibody was then blocked by overlaying the specimen with goat serum for 20 minutes at $37^{\circ} \mathrm{C}$. The blocking solution was poured off the slides and appropriate dilutions of the primary antibodies were applied to the sections and incubated at $37^{\circ} \mathrm{C}$ for 60 minutes. The primary antibody was washed off with TBS followed by 30-minute incubation with biotinylated link antibody and for 30 minutes with peroxidase labelled streptavidin. The staining was completed after a 10-minute incubation with AEC (Amino-ethylcarbazole) substrate. The sections were lightly counterstained with hematoxylin and cover slipped with glycergel.

\section{Results}

The sections of the biopsy specimens obtained at diagnosis revealed a very cellular marrow with little or no fat due to an infiltration by blast cells (Figures 1a \& 1b) in five of the six patients. In one patient the cellularity was less than $50 \%$. Erythroid precursors, mature granulocytes and metamyelocytes, and megakaryocytes were either markedly reduced in number or absent. Immediately following the intensive chemotherapy regimen (day 7) a considerable reduction in cellularity due mainly to the reduction in the blast cell population was evident. The cellular depletion continued with time and the marrow became increasingly hypocellular, grossly edematous with widely dilated sinus system (Figure $2 \mathrm{a}$ ). A population of cells which had accumulated fat droplets (young multi-loculated fat cells) was also noted (Figure 2b). These changes were accompanied by the appearance of increasing numbers of well differentiated lymphocytes, plasma cells, and macrophages. A few mast cells were also commonly seen at this stage. In most instances, the lymphocytes and plasma cells were diffusely scattered but mature plasma cells were also seen aligned along well-preserved small blood vessels as perivascular cuffs (Figure 3 ). 


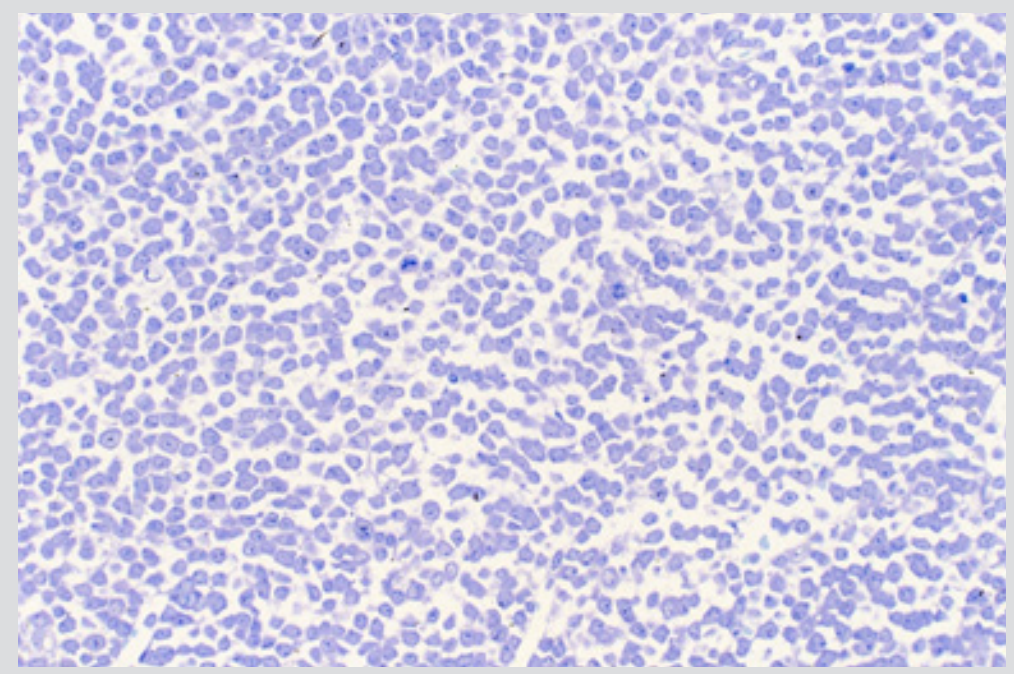

Figure 1a: BM biopsy section from a case of AML at diagnosis demonstrating highly cellular marrow with dense infiltration by immature blast cells. Note there are no fat cells. (Glycol-methacrylate, May-Grunewald-Giemsa stain).

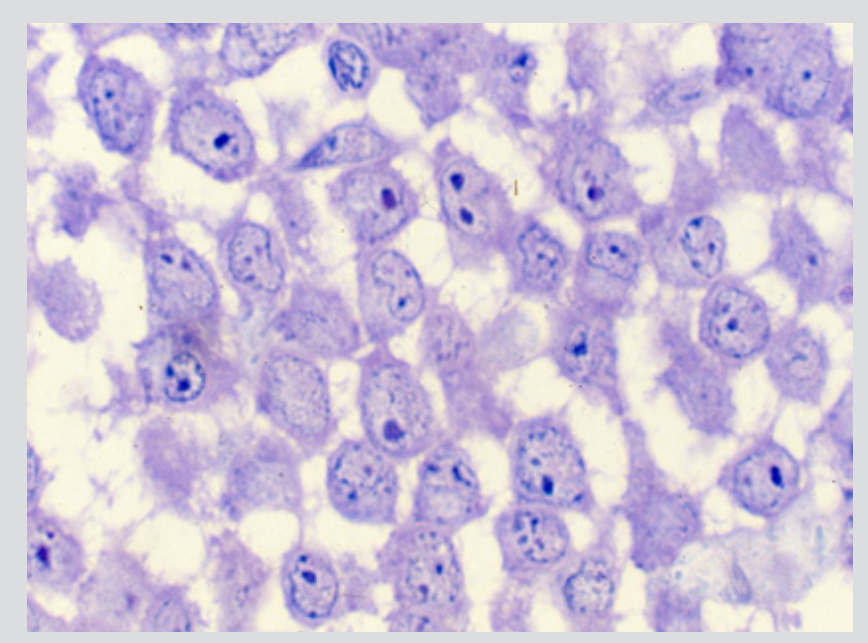

Figure 1b: BM biopsy section from the same case as figure 1a, higher magnification demonstrating the morphology of blast cells in improved detail. Note the presence of nucleoli in blast cells. (Glycol-methacrylate, May-Grunwald-Giemsa stain).

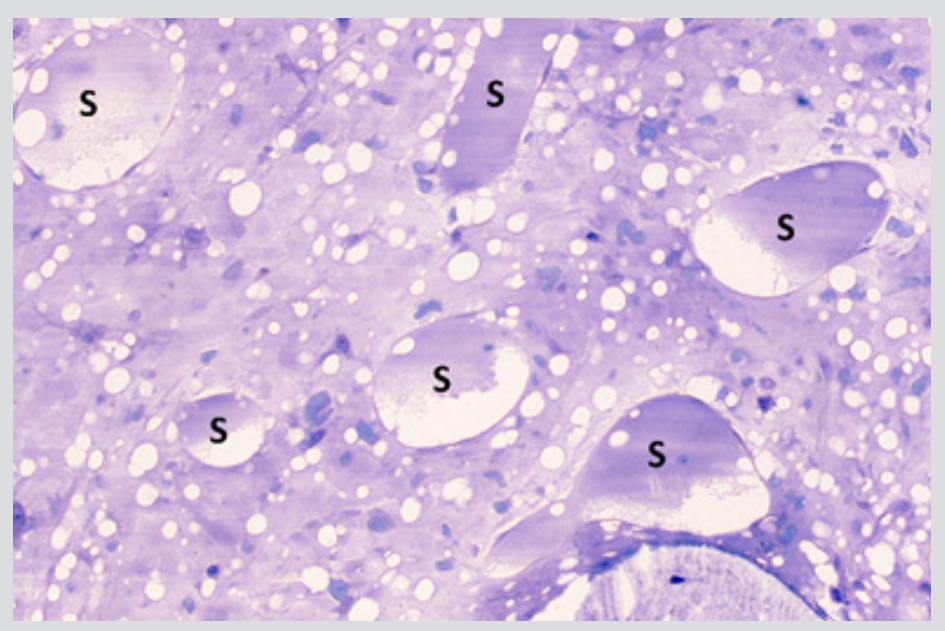

Figure 2a: BM biopsy section from a case of AML following the completion of intensive chemotherapy showing a hypocellular and grossly edematous marrow with widely dilated sinuses $(S)$. 


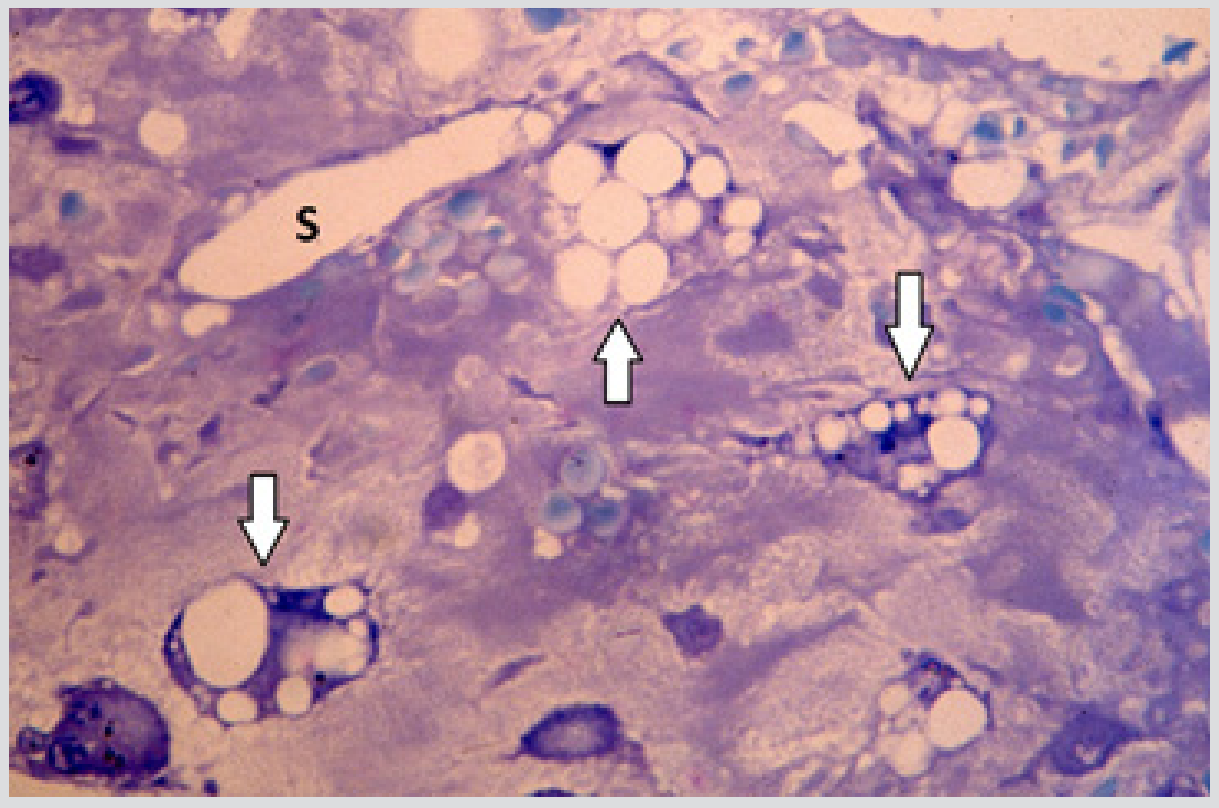

Figure 2b: BM biopsy section from the same case as in Figure 2a, another area of the section showing young multiloculated fat cells (arrows). (Glycol-methacrylate, May-Grunwald-Giemsa stain).

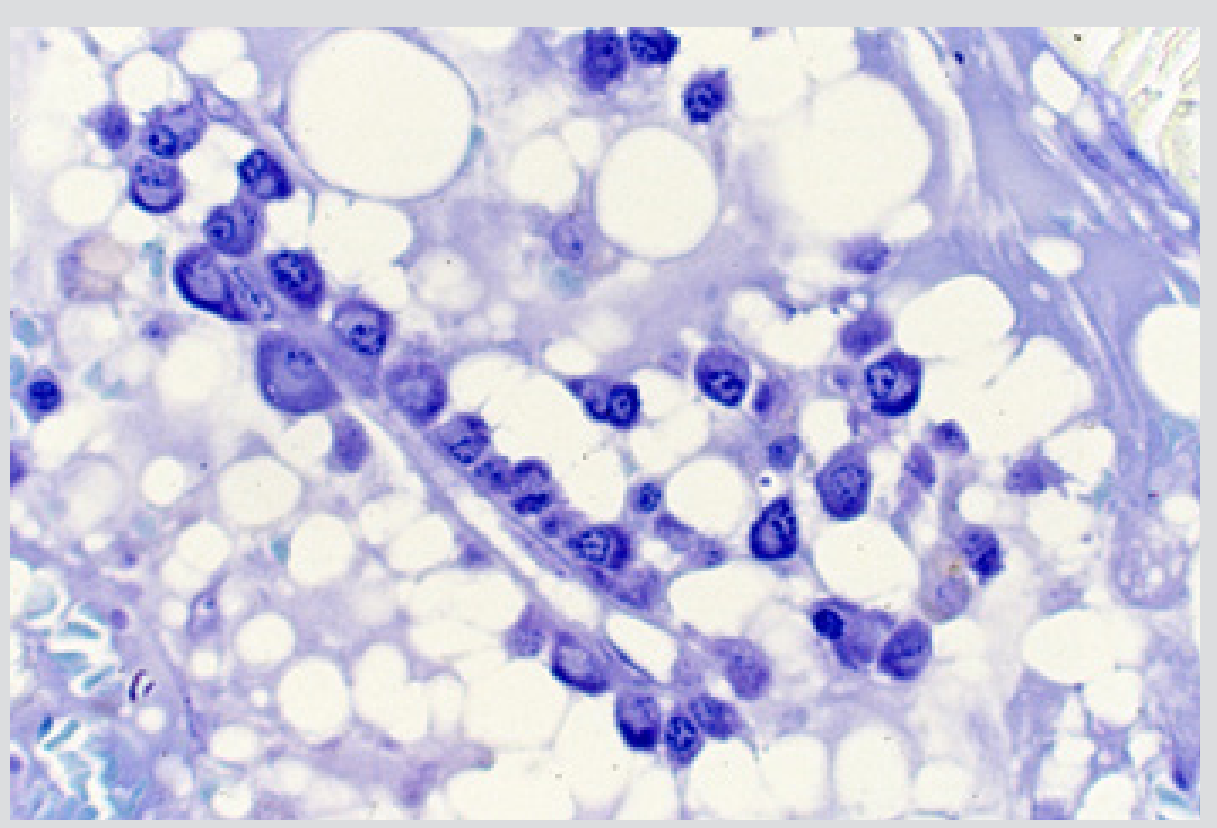

Figure 3: BM biopsy sections from cases of AML following intensive chemotherapy revealing plasma cells located around small blood vessels as perivascular cuffs. (Glycol-methacrylate, May-Grunwald-Giemsa stain).

The plasma cells were polyclonal as evidenced by the fact that some of them were stained for kappa and others were stained for lambda light chains with respective antibodies (Figures $4 \mathrm{a} \& 4 \mathrm{~b}$ ). The endosteal cells during this period were noted to be larger and more conspicuous with finely dispersed chromatin and single or multiple small nucleoli (Figure 5). Some of these apparently activated endosteal cells were seen to migrate into the surrounding acellular, edematous intertrabecular marrow space as fibroblastlike cells (Figure 6). Two to four weeks following the end of chemotherapy, thick margins of regenerating hematopoietic cells were seen closely adjacent to bony trabeculae (Figure 7), arterioles and arterial capillaries, marrow sinusoids and fat cells (Figure 8). These foci of regenerating hematopoietic cells were predominantly granulocytic, an observation which was confirmed by their positive reaction with the anti-myeloperoxidase antibody. A proportion of these cells were observed to migrate into the central intertrabecular marrow space where they formed colonies of more mature and differentiated hematopoietic cells. 


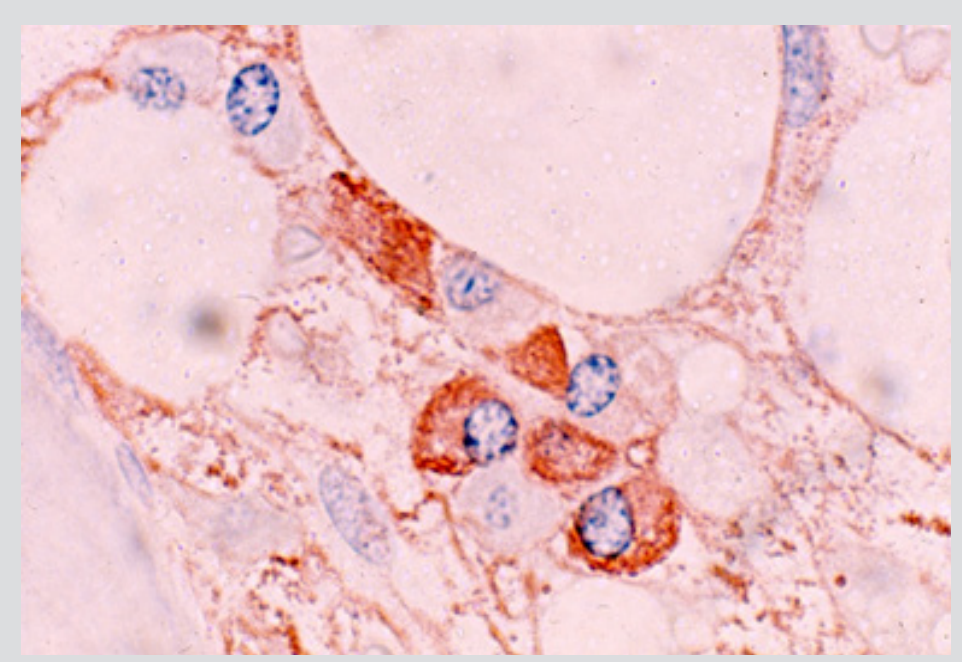

Figure 4a: BM biopsy sections from a case of AML immunologically stained for kappa light chains, showing kappa positive plasma cells (Glycol-methacrtlate, PAP/AEC/hematoxylin).

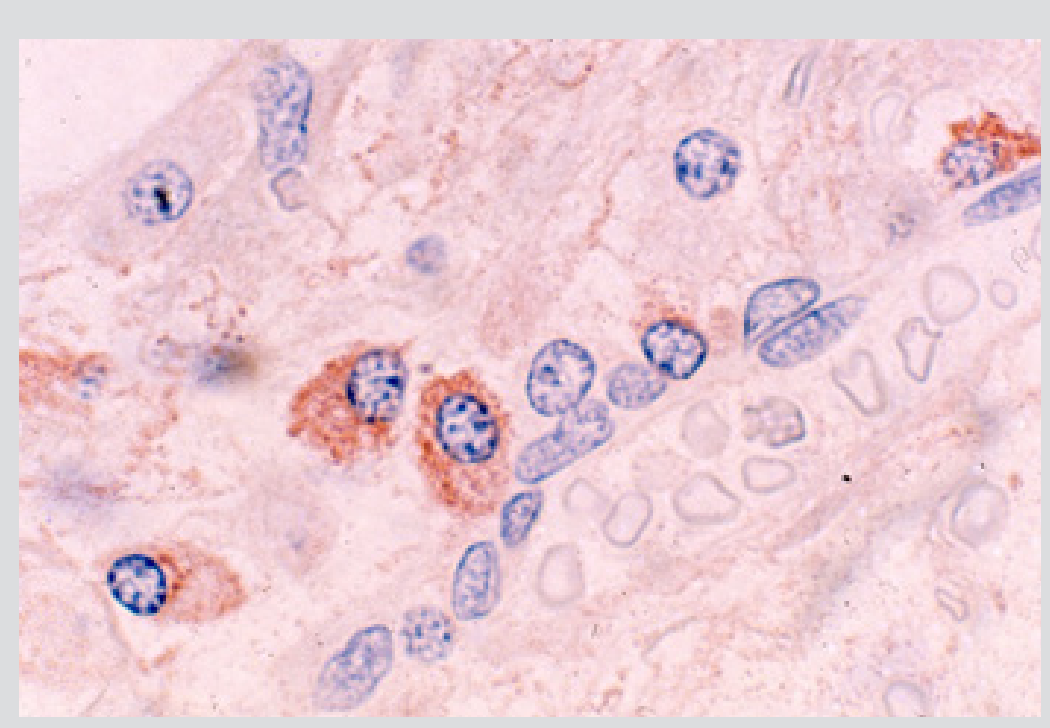

Figure 4b: BM biopsy sections from same case as in figure 4a immunologically stained for lambda light chains, showing lambda positive plasma cells (Glycol-methacrtlate, PAP/AEC/hematoxylin).

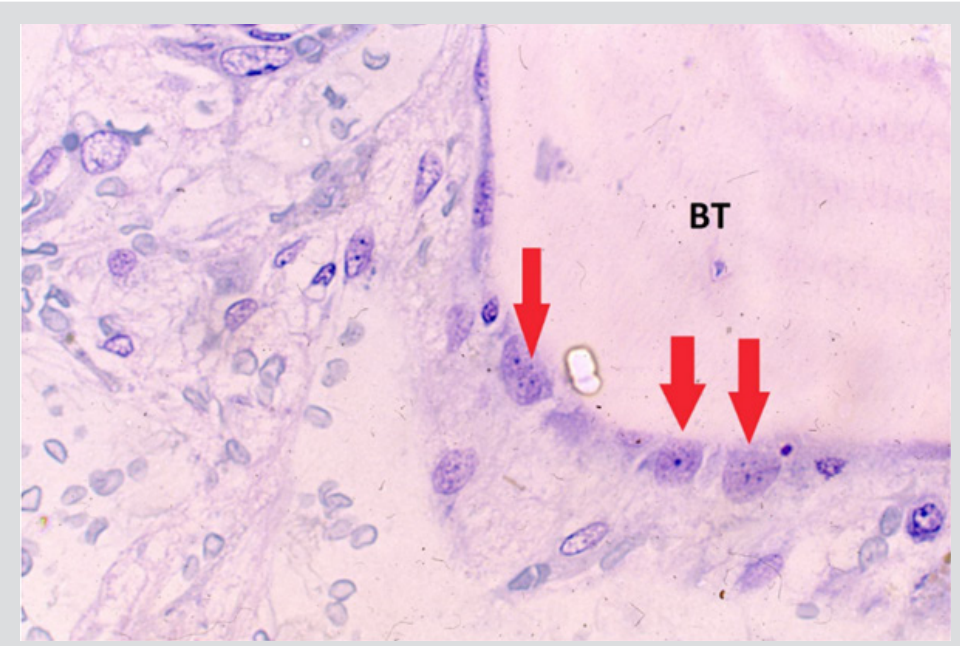

Figure 5: BM biopsy section from a case of AML following intensive chemotherapy showing swollen and apparently activated endosteal cells (arrows). Note bony trabecula (BT) at the upper right-hand corner of the photomicrograph. (Glycol-methacrylate, May-Grunwald-Giemsa stain). 


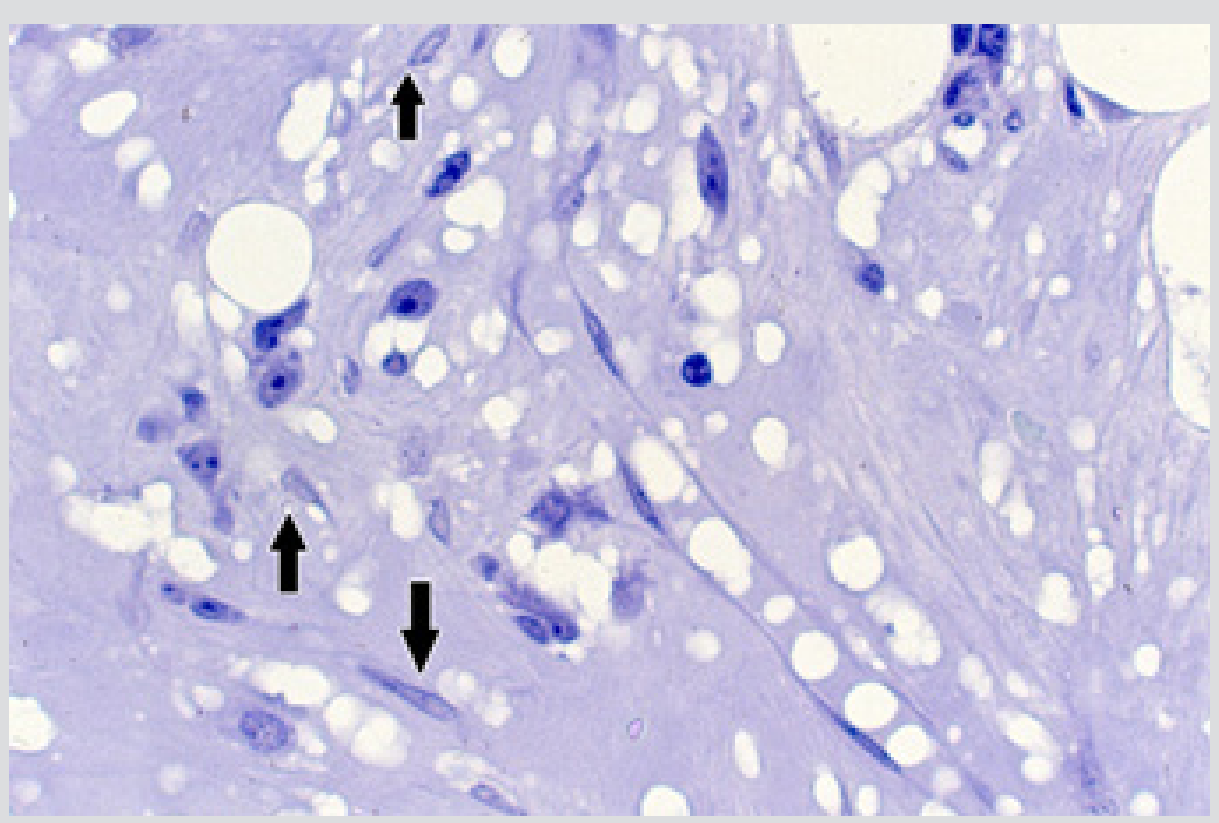

Figure 6: BM biopsy section from the same case as in figure 5 demonstrating fibroblast-like cells (arrow) in the central hypocellular and edematous inter-trabecular marrow space. (Glycol-methacrylate, May-Grunwald-Giemsa stain).

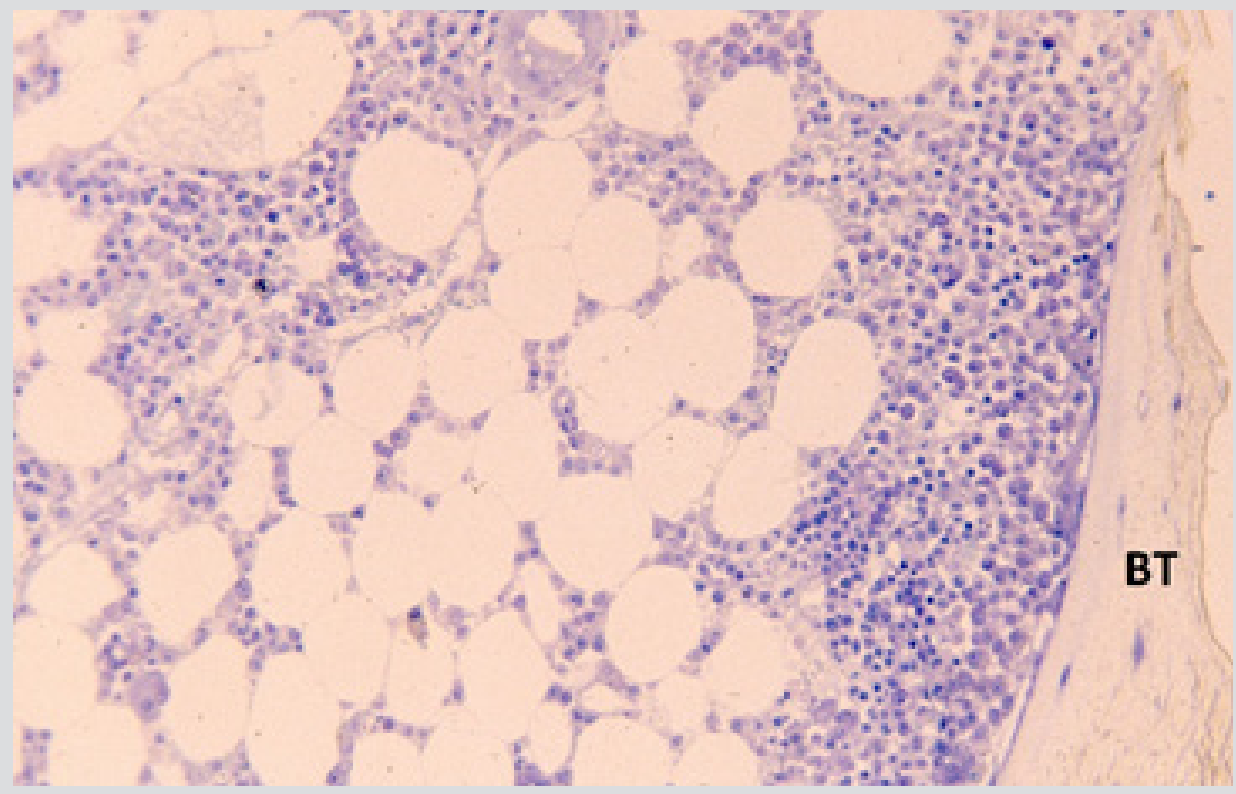

Figure 7: BM biopsy sections from a case of AML 4 weeks following the end of chemotherapy showing paratrabecular granulopoiesis. BT - bony trabecula. (Methyl-methacrylate, May-Grunwald-Giemsa stain).

The nuclear features of the activated 'endosteal' or 'fibroblastlike' cells and the multiloculated fat cells were very similar in their morphological appearance suggested that they might have originated from the peripheral flattened endosteal cells. The sections of biopsy specimens obtained five to eight weeks following chemotherapy showed active hematopoietic marrow in which all three cell lines of hematopoiesis were represented. But here again the hematopoiesis was dominated by a proliferation of cells belonging to the granulocytic cell line as evidenced by morphology
(Figure 9) and the large number of myeloperoxidase positive cells. Even at this stage where hematopoiesis was quite advanced the characteristic paratrabecular and perivascular localization of these hemic cells continued to be evident. A considerable increase in the number of eosinophilic granulocytes were also observed in MGG stained sections of most of the biopsy specimens at this time verified by staining with antieosinophilic peroxidase monoclonal antibody staining (Figure 10). 


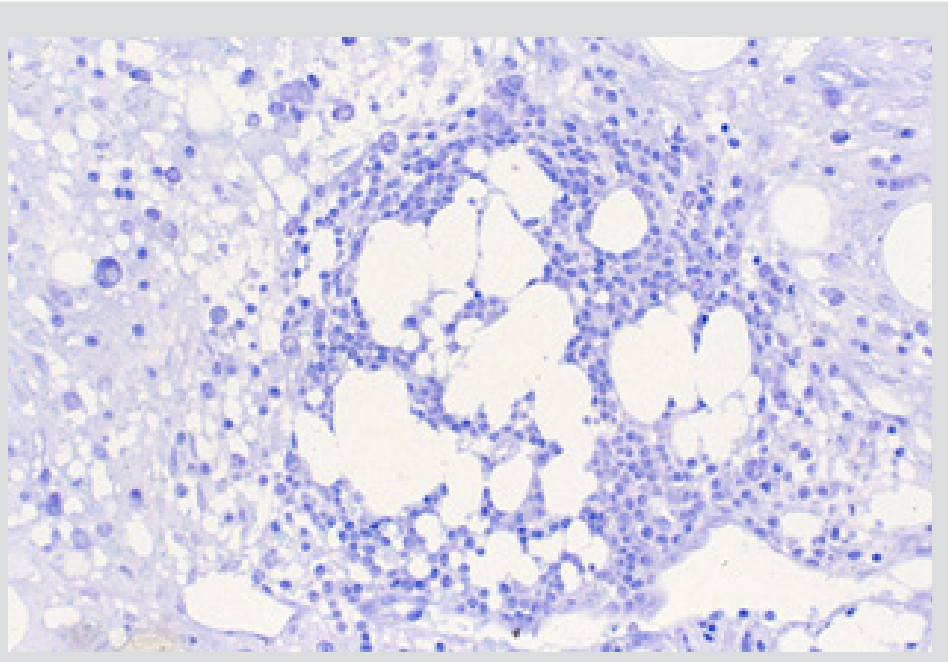

Figure 8: BM biopsy section from a case of AML 4 weeks following the end of chemotherapy showing hematopoiesis around the fat cells. (Glycol-methacrylate, May-Grunwald-Giemsa stain).

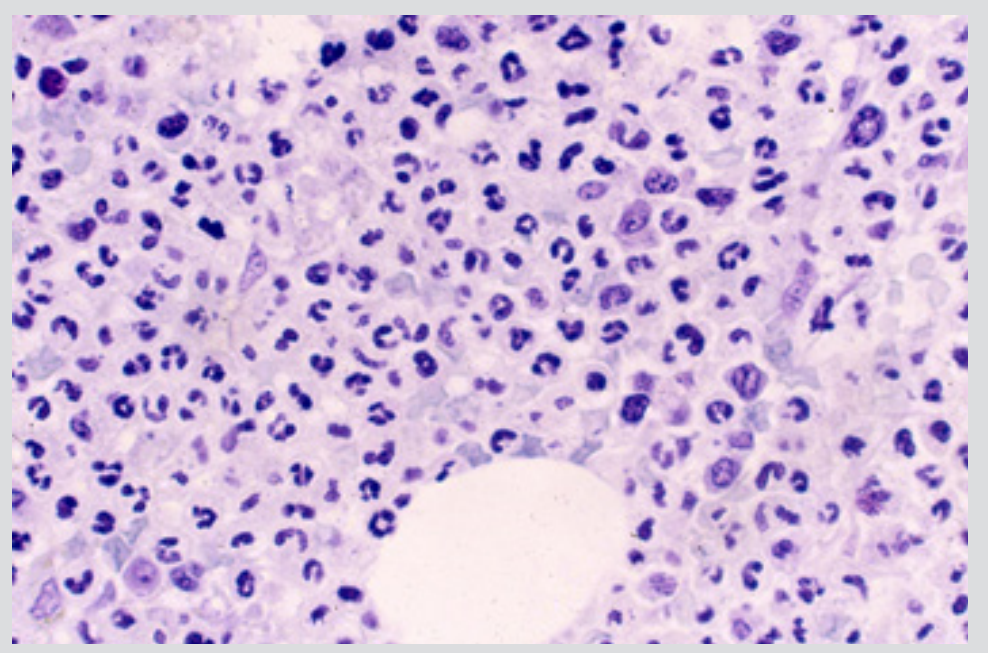

Figure 9: BM biopsy section from a case of AML 6 weeks following the end of chemotherapy demonstrating marked granulo-cytic proliferation (Glycol-methacrylate, May-Grunwald-Giemsa stain).

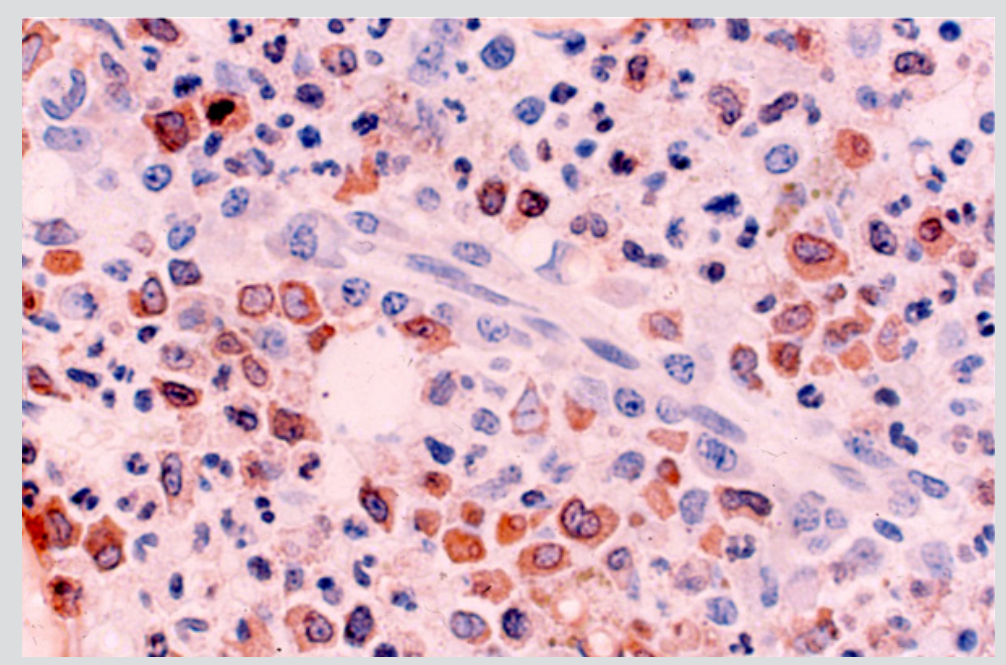

Figure 10: BM biopsy section from a case of AML 6 weeks following the cessation of chemotherapy showing clusters of eosinophilic granulocytes around a small blood vessel. The elongated endothelial cells can be clearly seen. The section was stained with anti-eosinophilic peroxidase antibody. (Glycol-methacrylate, PAP/AEC/hematoxylin). 


\section{Discussion}

Although the effects of intensive chemotherapy and cytokine treatment on hematopoiesis have been studied extensively in human, most if not all of these investigations were carried out on peripheral blood and/or bone marrow obtained by aspiration. Unfortunately, sectioned trephine bone marrow biopsies were not conventionally used for this purpose even though it is well known that a solid core BM biopsy avoids the sampling errors inherent in the technique of BM aspiration. In addition, the spatial distribution of the hematopoietic cell populations and stromal components are well preserved and remain intact in sections and thus they are retained for analysis. In the present study we focused on the effects of combined intensive chemotherapy and Rh G-CSF treatment on the bone marrow of patients with AML using plastic embedded BM biopsies. Plastic embedding was selected because of the advantages this technique offers over conventional method of decalcification and paraffin embedding. In addition, the interface between bone and marrow and marrow and blood vessels are also particularly well preserved in plastic embedded BM biopsy sections.

Thus, the endosteal and perivascular regions (regenerative and proliferative loci of marrow) [15] can be microscopically evaluated. Another advantage is that/ immunohistology techniques can be applied to plastic (GMA) embedded specimens. Thus, the antigenic make up of various cellular populations including those which are focally distributed or firmly anchored within "paratrabecular" and "perivascular" regions of the marrow can be assessed in their natural environment. Immediately following therapy and during the hypoplastic phase increasing numbers of inflammatory cells especially lymphocytes, plasma cells and macrophages were regularly seen in the sections of BM from these patients. The possibility that this latter response was due to the effect of G-CSF was ruled out because of the fact that a similar response was invoked in some of our AML patients that were subjected to intensive chemotherapy but without any associated cytokine treatment [16]. The significance of the appearance of these cells in post-therapy marrow remains uncertain. The ability of lymphocytes and macrophages to stimulate immune responses is well known $[17,18]$.

Whether these cells are responsible in mounting an immune response in these severely neutropenic and perhaps somewhat immunocompromised patients may need further investigation. The macrophages might also be present as scavenger removing dead or dying cells remaining after the chemotherapy. The presence of large numbers of macrophages with prominent dark staining debris is well recognized in leukemic patients in complete remission. Following the cessation of chemotherapy and during the early period of hematopoietic regeneration, the cellular proliferation was almost exclusively localized near the bony trabeculae where myeloblasts, promyelocytes and metamyelocytes predominated and formed thick bands of cells along the endosteal surface of bone trabeculae. In some cases, hematopoietic cells, (mostly granulocytic as positively identified by the MPO reaction) were also localized near the marrow sinusoids and small blood vessels. The role of endosteal and vascular endothelial cells in fetal, normal, diseased and stressed (post-chemotherapy, post-marrow curettage) conditions has been intensively studied and reported elsewhere $[15,19]$. The present finding only confirms our previous observation that characteristic areas of regeneration and proliferation exist within the bone marrow. These areas, we believe, are the rich sources of hematopoietic stem cells. The biological effects of various cytokines as G-CSF presumably are maximally expressed here.

In all patients Rh G-CSF appeared very effective in inducing granulocytic proliferation during the early (2-4 weeks) and late (5-8 weeks) phases of hematopoietic regeneration. In all cases a proliferation of eosinophilic granulocytes was observed at 5-8 weeks following the end of chemotherapy. There was no accompanying eosinophilia in the peripheral blood. The ability of Rh G-CSF to increase polymorphonuclear neutrophils is well documented [20] but its ability to increase eosinophil granulocytic proliferation in the marrow has not yet been recognized. In fact, it has been reported that G-CSF neither affects differentiation nor proliferation of human eosinophilic HL-60 cells [21]. The reason for their presence in such increased numbers and the delay (5-8 weeks) in their appearance remains unclear. The stromal changes observed after treatment included an increase in the number of fibroblasts-like cells. The presence of young multilocular fat cells and large uniform unilocular mature fat cells was also noted [16]. It has been shown that fat cells play an important role in the maintenance of the hematopoietic stem cells and their growth in the mouse marrow stromal cultures [22]. In the Dexter culture system granulocytic proliferation has been shown to take place preferentially in the regions containing fat cells, particularly on the periphery of such areas where the cells are in an early stage of lipid accumulation [23].

Although in AML patients (treated with intensive chemotherapy only) hematopoietic regeneration was observed mostly within areas manifesting unilocular fat cells [16], the association of hematopoietic foci to areas containing either immature or mature fat cells was not readily obvious in our AML patients who were simultaneously treated with Rh G-CSF. In these cases, the hypoplastic stage, characterized by edema and dilated sinuses, was quickly succeeded by a period of rapid regeneration of hematopoietic cells anatomically related to paratrabecular and perivascular regions and also near the structured fat. The close association of regenerating hemic cells with endosteum and endothelium suggests that the latter play an important role in hematopoietic recovery. It has been shown that hematopoietic progenitor cell proliferation and differentiation within the bone marrow depends, at least in part, on an appropriate microenvironment provided by a stromal cell population comprised of fibroblasts, endothelial cells adipocytes and macrophages [24]. Bone marrow stromal cells are known to provide necessary cytokines and extracellular matrix components required for the proliferation and maturation of circulating blood cells. 


\section{Acknowledgement}

This research was conducted in collaboration with Geoffrey $\mathrm{P}$ Herzig, M.D. (deceased) when the author was a research associate at Roswell Park Cancer institute, Buffalo, New York.

\section{References}

1. Clark S, Kamen R (1987) The human hematopoietic colony stimulating factors. Science 236(4806): 1229-1237.

2. Nicola NA (1989) Hematopoietic cell growth factors and their receptors. Annu Rev Biochem 58: 45-77.

3. Metcalf D (1989) The molecular control of cell division,differentiation, commitment and maturation in hematopoietic cells. Nature 339(6219): 27-30.

4. Heyworth CM, Vallance SJ, Whalton A, Dexter TM (1990) The biochemistry and biology of the myeloid haemopoietic growth factors. J cell Sci Suppl 13: 57-74.

5. Glaspy JA, Golde DW (1990) Clinical trials of myeloid growth factors. Exp Hematol 18: 1137-1141.

6. Metcalf D (1991) Control of granulocytes and macrophages: Molecular, cellular, and clinical aspects. Science 254(5031): 529-533.

7. Groopman JE, Molina JM, Scadden DT (1989) Hematopoietic growth factors. Biology and clinical applications. N Eng J Med 321(21): 14491459.

8. Sieff CA (1990) Biology and clinical aspects of the hematopoietic growth factors. Annu Rev Med 41: 483-496.

9. Orazi A, Cattoretti G, Schiro R, Siena S, Bregni M, et al. (1992) Recombinant human interleukin-3 and recombinant human granulocyte-macrophage colony-stimulating factor administered in vivo afterhigh-dose cyclophosphamide cancer chemotherapy: effect on hematopoiesis and microenvironment in human bone marrow. Blood 79(10): 2610-2619.

10. Baer MR, Bernstein SH, Brunetto VI, Minderman H, Rustum Y, et al. (1993) In vivo recombinant human G-CSF priming in previously untreated acute myeloid leukemia. Blood 82: 1298 .

11. Jamshidi K, Windschitl HE, Swaim WR (1971) A new biopsy needle for bone marrow. Scand J Haematol 8(1): 69-71.

12. Islam A (1982) A new bone marrow biopsy needle with core securing device. JClin Pathol 35(3): 359 -364

13. Islam A, Henderson ES (1987) Glycol methacrylate embeddding for light microscopy. I. Enzyme histochemistry on sections of undecalcified marrow core. J Clin Pathol 40(10): 1194-1200.

\section{ISSN: 2574-1241}

\section{DOI: 10.26717/BJSTR.2019.20.003434}

\section{Anwarul Islam. Biomed J Sci \& Tech Res}

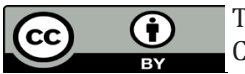

This work is licensed under Creative Commons Attribution 4.0 License

Submission Link: https://biomedres.us/submit-manuscript.php
14. Islam A, Archimbaud E, Henderson ES, Han T (1988) Glycol methacrylate (GMA) embedding for light microscopy. II. Immunohistochemical analysis of semithin sections of undecalcified marrow cores. J Clin Pathol 41(8): 892-896.

15. Islam A, Glomski C, Henderson ES (1990) Bone lining (endosteal) cells and hematopoiesis: A light microscopic study of normal and pathologic human bone marrow in plastic-embedded sections. Anat Rec 227(3): 300-306.

16. Islam A, Catovsky D, Galton DAG (1980) Histological study of bone marrow regeneration following chemotherapy for acute myeloid leukaemia and chronic granulocytic leukaemia in blast transformation. Br J Haematol 45(4): 535-540.

17. Geppert TD, Lipsky PE (1989) Antigen presentation by cells that are not of bone marrow origin. Reg Immunol 2(1): 60-71.

18. Germann T, Mattner F, Partenheimer A, Schmitt E, Reske Kunz AB, et al. (1992) Different accessory function for TH1 cells of bone marrow derived macrophage cultured in grtanulocyte macrophage colony stimulating factor or macrophage colony stimulating factor. Int Immunol 4(7): 755-764.

19. Islam A, Glomski C, Henderson ES (1991) Endothelial cells and hematopoiesis: a light microscopic study of fetal, normal, and pathologic human bone marrow in plastic-embedded sections. Anat Rec 233(3): $440-452$.

20. Bensinger WI, Price TH, Dale DC, Appelbaum FR, Clift R, et al. (1993) The effects of daily recombinant human granulocyte colony-stimulating factor administration on normal granulocyte donors undergoing leukapheresis. Blood 81(7): 1883-1888.

21. Fabian I, Lass M, Kletter Y, Golde DW (1992) Differentiation and functional activity of human eosinophilic cells from an eosinophil HL-60 subline: response to recombinant hematopoietic growth factors. Blood 80(3): 788-794.

22. Dexter TM, Allen TD, Lajtha LG (1977) Conditions controlling the proliferation of haemopoietic stern cells in vitro. J Cell Physiol 91(3): 335- 344.

23. Allen TD. Ultrastructural aspects of in vitro haemopoiesis. The second symposium of the British Society for Cell Biology on Stem Cells and Tissue Homeostasis p. 230.

24. Singer JW, Keating A, Wight TN (1985) The human hematopoietic microenvironment, in Hoffbrand AV (eds.). Recent Advances in Hematology. New York, USA, p. 1.

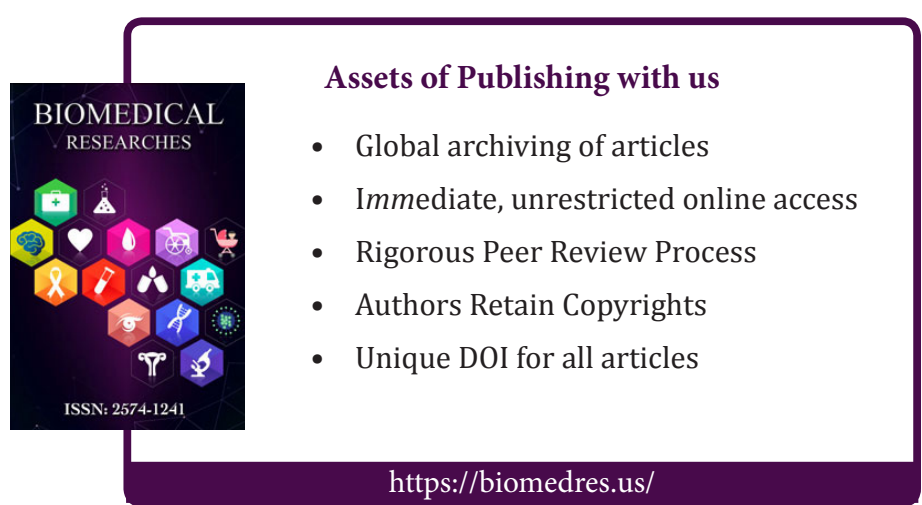

